\title{
Convex hull as a heuristic
}

\author{
Naama Katzin ${ }^{a, b *}$, David Katzinc ${ }^{c}$, Adi Rosen ${ }^{d}$, Avishai Henik ${ }^{a, b}$ \& Moti Salti ${ }^{b}$

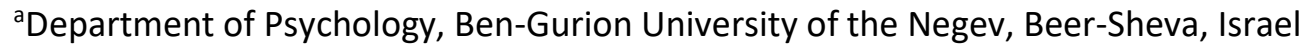

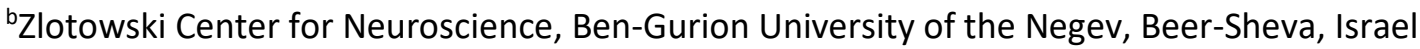 \\ 'Department of Plant Sciences, Wageningen University, Wageningen, The Netherlands \\ ${ }^{\mathrm{d} C N R S}$ and Universite Paris Diderot
}

Physical properties such as area and convex hull (the smallest polygon containing all objects) have been suggested to be the building blocks of numerical cognition ${ }^{1}$. Many studies measured the effect of physical properties on numerical comparison tasks ${ }^{2,3}$, but it was not generalized to other numerical tasks. Moreover, no cognitive mechanism underlying the influence of physical properties on numerical cognition (that is, not only numerical comparisons) was suggested. Here we put forward a parsimonious model that demonstrates how physical properties influence numerical cognition, and specifically, enumeration. Using a computer simulation, we show that the shape of the convex hull can be used to compress information regarding numerosity. Convex hull predictability changes with increasing numerosity, creating two qualitatively different ranges corresponding to the behavioral enumeration literature-the subitizing range (1-4) in which numbers are enumerated accurately, and the estimation range (5 and up) in which enumeration accuracy drops ${ }^{4}$. In two behavioral experiments, we show that the shape of the convex hull directly influences participants' numerical estimation. We conclude that the shape of the convex hull is used as a heuristic for enumeration. This rough estimation mechanism enables other fine-grained numerical abilities.

In 1871, Stanley Jevons published his 'Nature' report on how many objects the mind can perceive in a single mental beat of attention. To this end, he repeatedly threw a handful of black 
beans on a tray and tried to estimate their number. After each throw, he recorded the actual number of beans on the tray and his quick estimation. A thousand trials revealed he was $100 \%$ accurate in quantities up to 4 , but at 5 accuracy deteriorated. These results led him to conclude that 4 is the maximal number of objects that can be perceived at once ${ }^{5}$. This report is the first of many that described the human ability to quantify.

Enumeration is divided to subitizing (1-4), and estimation (5 and up). Numerosities in the subitizing range are enumerated accurately and reaction time (RT) increases by $50-80$ ms per additional item. Numerosities above four are counted if there is enough time, in which case RT increases by $\sim 200$ ms per additional item. If time does not allow for counting, numerosities are estimated quickly with a cost in accuracy ${ }^{4}$. Despite the consensus on this division, it remains unclear whether subitizing and estimation rely on two distinct mechanisms or if they are the product of a single mechanism that operates with varying efficiency ${ }^{6}$.

What might explain the restricted range of subitizing? One line of explanations suggests that domain-general functions such as attention and short-term memory restrict the subitizing range $^{7,8}$. However, these cannot account for the increase in RT within the subitizing range ${ }^{6}$. Alternatively, it has been suggested that subitizing relies on a pattern recognition mechanism. When items are presented in a familiar pattern (i.e. canonically), the pattern is quickly and efficiently translated into numerosity ${ }^{9}$. However, in real life, objects are not necessarily arranged canonically; on the contrary, canonical arrangements are relatively rare. Moreover, an imaging study demonstrated that pattern recognition is not exclusive to canonical arrangements or to the subitizing range. The behavioral results in this study did not find a difference in RT between canonical and random arrays in the subitizing range. In addition, brain areas associated with pattern recognition were activated in an enumeration task regardless of range ${ }^{6}$. 
It seems that canonical arrangements encompass only a small portion of the phenomenon. However, this might suggest that people rely on spatial arrangements to assess numerosity by learning the statistical distribution of possible arrangements. The vast number of possible arrangements makes it unlikely and uneconomical to learn them all. Relying on spatial arrangement would be more plausible if it could exploit a single property that can downsize the number of possible arrangements while enabling distinction between numerosities. A good candidate for such a property is the shape of the convex hull $(\mathrm{CH})$. The $\mathrm{CH}$ of a finite set of dots is the smallest polygon containing all dots and all straight lines between any two dots (see Figure 1A). This polygon can be characterized by its shape or number of vertices (e.g. 3 (triangle), 4 (quadrilateral), etc.). The shape of the $\mathrm{CH}$ is a lower resolution version of any array of objects, as it uses the minimal number of objects (dots) that contain the entire array. Interestingly, five and six dots on a die are two distinct canonical shapes in the pattern recognition theory, however, their $\mathrm{CH}$ is similar - a quadrilateral (see Figure 1B).

A

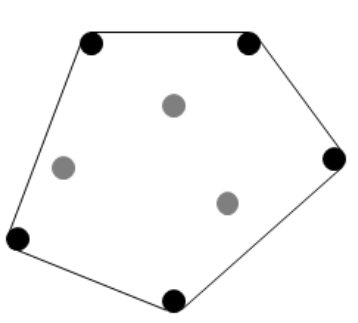

B

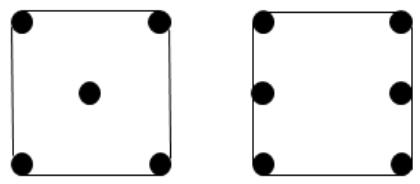

Figure 1 (A) A set of points and its convex hull. Convex hull vertices are black, interior dots are grey. (B) Convex hull of five and six dots, as presented on a die.

Consequently, we aimed to examine the extent to which the shape of the $\mathrm{CH}$ (i.e. number of vertices) predicts numerosity of a given set: if an unknown number of dots $(n)$ is randomly scattered over a region, how will knowing the shape of the $\mathrm{CH}$ ( $k$-number of vertices) help in estimating the total number of dots $(P(n \mid k))$ ? We were interested in the way the information $\mathrm{CH}$ 
holds about numerosity changes with quantity, and if these changes correspond to the documented ranges of enumeration (namely, subitizing and estimation).

This question relates to an open problem in the field of geometric probability, first posed by Sylvester ${ }^{10}$ and formally stated as follows: if $n$ points are chosen independently with uniform probability on a given region, what is the probability that the $\mathrm{CH}$ of these points is a polygon with exactly $k$ vertices $(P(k \mid n))$ ? The answer to this question depends on the shape of the given region; in the case of a rectangular region, it was solved for certain values of $\mathrm{k}\left(\mathrm{e} . \mathrm{g} . k=3^{11}, k=n^{12}\right.$ ), but generally the answer remains open. Nevertheless, the unknown values may be approximated empirically using a Monte Carlo simulation, similar to Jevons's experiment.

Accordingly, the probabilities $P(k \mid n)$ were estimated using a Monte Carlo simulation for every number $\mathrm{n}$ between 3 and 30. Table 1 depicts the calculated and simulated probabilities. Our results show that the probability that all dots are vertices of the $\mathrm{CH}$ (i.e. $k=n$ ) drops drastically at five (a drop from 0.6944 at four to 0.3403 at five). In fact, starting at $n=5$, the case of $k=n$ is no longer the most probable. Importantly, we wanted to know how predictive $k$ is of $n$ (i.e. $P(n \mid k)$ ). That is, given that exactly $\mathrm{k}$ points are on the convex hull, we ask what the probability is that the number of total points is exactly $\mathrm{n}$. The answer to this question depends on how the number of points is chosen. This can be seen by the use of Bayes' theorem:

$$
\text { (1) } P(n \mid k)=\frac{P(k \mid n) P(n)}{P(k)}
$$

Here, the number $\mathrm{k}$ is fixed, so $P(k)$ may be viewed as a normalizing constant, that is, a scaling factor ensuring that the probabilities $P(n \mid k)$ are between 0 and 1 . However, the choice of $P(n)$ strongly influences the values $P(n \mid k)$. For instance, choosing $P(n)$ as a uniform distribution, (i.e. assuming all numerosities $n \geq k$ are equally probable) gives for every fixed $k, P(n \mid k) \propto P(k \mid n)$ (i.e. $P(n \mid k)$ is directly proportional to $P(k \mid n))$. Unfortunately, it is impossible to assume that all 
numerosities greater than $\mathrm{k}$ are equally probable, since a discrete uniform probability on an infinite number of points does not exist; it follows that if a uniform probability is assumed, we must also assume an upper bound on the number of points.

Nevertheless, choosing a uniform probability for $P(n)$ gave qualitatively similar results, no matter what the choice of an upper bound was. While we cannot calculate $P(n \mid k)$ exactly, the relation $P(n \mid k) \propto P(k \mid n)$ allows us to know, given $\mathrm{k}$, which n's are more probable, and their relative likelihood. For $k=3$ and $k=4$, the most probable numerosity is $n=k$. For these cases, the ratio between the most probable and second most probable $n$ is 3.27 and 1.25, respectively (i.e. $\frac{1}{0.3056}$ and $\left.\frac{0.6944}{0.5556}\right)$. For $k=5$, not only is it less likely that $n=k$, but the ratio between the most and second most probable $\mathrm{n}$ is only 1.02 (i. e. , $\frac{0.4815}{0.4698}$, see Table 1 ). As $k$ increases, this ratio approaches 1. Hence, for $n \leq 4, \mathrm{CH}$ highly predicts a specific numerosity; otherwise, $\mathrm{CH}$ predicts an expanding range of numerosities. Similar results were found when the dots were randomized on a disk rather than a rectangle.

The simulation demonstrates that the shape of the convex hull is predictive of numerosity. Its predictability up to four is quite accurate, and above four it predicts an expanding range of numerosities. One of the predictions of these results is that the number of vertices on the convex hull should predict estimation of numerosity. Namely, when the number of vertices on the convex hull is the most likely amount, that is, the most probable shape, estimations should be most accurate. A higher or lower number of vertices on the convex hull should yield over or under estimations, respectively. For example, for numerosity 12, we would expect the most accurate enumerations with 6 vertices on the convex hull, under-estimations when there are less than 6 vertices on the convex hull, and over-estimations for more than 6 vertices on the convex hull. 


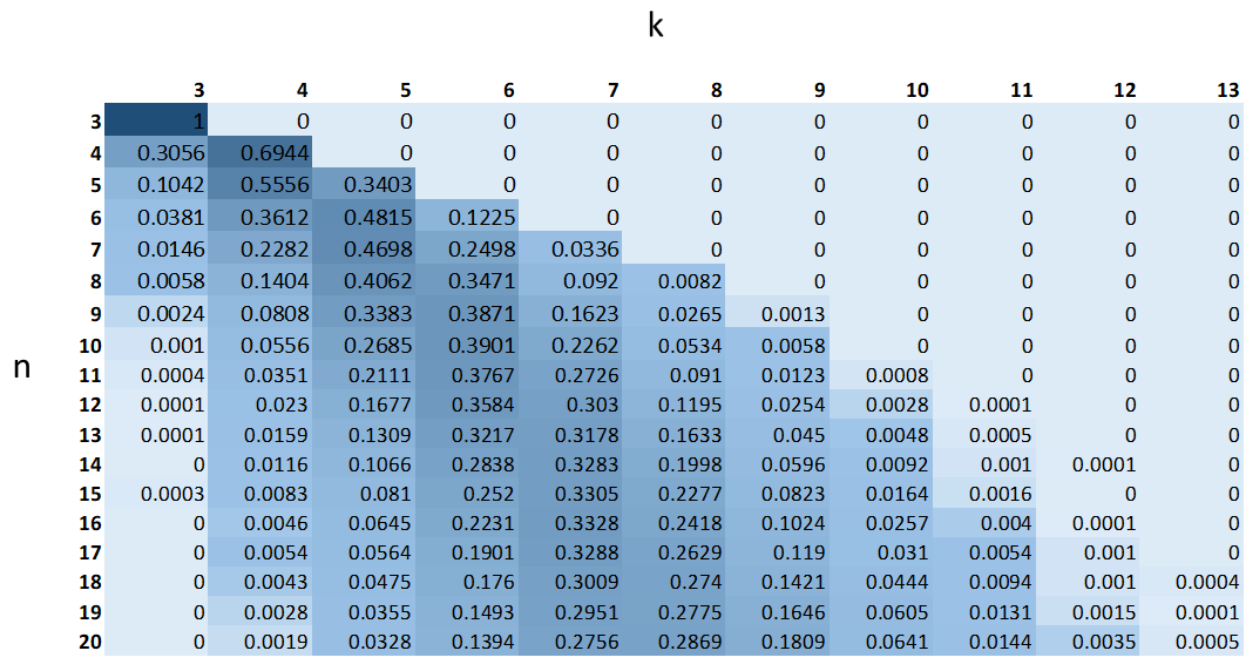

Table 1 The simulated probabilities of different patterns. Each row in the table represents all possible arrangements for a given numerosity and sums to $1 . n$ - total numerosity in the array. $k$ - number of vertices on the convex hull.

To investigate this prediction, we conducted two experiments in which the number of vertices on the convex hull was manipulated. Participants (total $N=52$ ) were instructed to estimate the number of dots in an array of dots that appeared briefly on the screen. In the first experiment, the numerosities 7-10 appeared with 3-7 vertices on the convex hull. We found a significant main effect for total numerosity, $F(3,45)=160.48, p<.001, \eta_{p}^{2}=.91$, so that estimations were higher as numerosity was higher. In addition, a main effect for number of vertices on the convex hull was found, $F(4,60)=5.9, p<.001, \eta_{p}^{2}=.28$. Namely, estimations were higher as the number of vertices on the convex hull grew (see Figure 2). The 2-way interaction between total numerosity and number of vertices on the convex hull was significant as well, $F(12,180)=1.98, p<.05, \eta_{p}^{2}=.12$.

In the second experiment, we had several goals. First, we wanted to replicate the results of the first experiment and expand it to larger numerosities. Second, we wanted to make the arrays more ecological, so we varied the size of the dots. Third, in the first experiment we created 
a factorial design by keeping the number of vertices on the convex hull similar for all numerosities. This design had two limitations. First, it constrained the number of numerosities. Second, it did not control for the probability of appearance of each condition. For example, for numerosity 7 , the probability for the most probable configuration is 0.4698 ( 5 vertices on the convex hull), and the least probable is 0.0146 ( 3 vertices on the convex hull). For numerosity 10 , the values are 0.3901 ( 6 vertices on the convex hull) and 0.001 ( 3 vertices on the convex hull), respectively. Accordingly, in this experiment we used numerosities 7-20. For each numerosity, we chose three different patterns that varied in the number of vertices on the convex hull. The 'standard' pattern was the most probable pattern. For example, for the numerosity 12 , the most probable pattern has 6 vertices on the convex hull (see Table 1). The 'low' and 'high' patterns are the patterns that are at least $30 \%$ less likely to appear compared to the standard, with a lower or higher number of vertices on the convex hull, respectively. For example, for the numerosity 12 , the 'low' pattern has 4 vertices on the convex hull, and the 'high' pattern has 9.

We found a significant main effect for total numerosity, $F(13,455)=245.6, p<.001, \eta_{p}^{2}$ $=.87$, so that estimations were higher as numerosity was higher. A main effect for number of vertices on the convex hull was significant as well, $F(2,70)=10.84, p<.001, \eta_{p}^{2}=.24$. Namely, estimations were higher as the number of vertices on the convex hull grew (see Figure 2). In addition, the interaction was significant, $F(26,910)=2.81, p<.001, \eta_{p}^{2}=.07$. 
Experiment 1

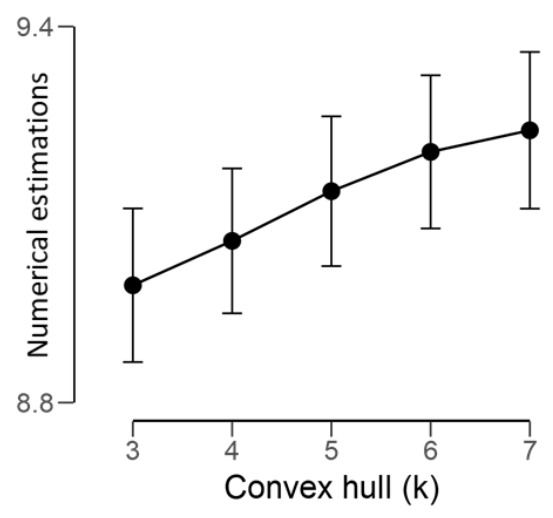

Experiment 2

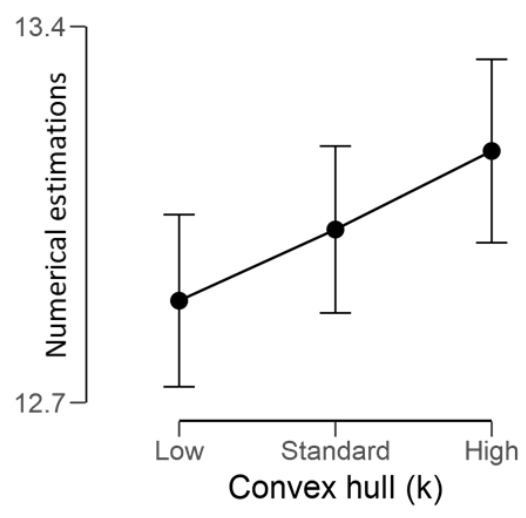

Figure 2 Main effect of number of vertices on convex hull for both experiments.

The results of the simulation and the behavioral experiments suggest that the number of vertices on the convex hull could be the underlying mechanism for perceiving and processing numerosities. Up to four, the shape of the convex hull predicts a specific numerosity accurately. Above four, the shape of the convex hull can predict an expanding range of numerosities. Redescribing the uniform distribution of numerosities as the distribution of number of vertices on the convex hull, yields a distribution that resembles the psychophysical curve of enumeration: subitizing, counting and estimation. We suggest that through the process of statistical learning, humans learn the correlation between number of vertices on the convex hull and numerosity and use it to enumerate. In daily life, we encounter a great deal of stimuli. Processing all of them is not only a huge cognitive load but also inefficient as some stimuli are more important than others are. The human cognitive system has many ways of dealing with the vast amount of information. For example, the attention system reduces the number of stimuli by focusing on a subset of them ${ }^{13}$. Another mechanism that helps ease cognitive load in the decision-making process is heuristics. A heuristic is a cognitive shortcut that relies on a subset of the information for making 
a decision, without damaging performance ${ }^{14}$. We suggest that the shape of the convex hull is utilized as a heuristic for enumeration.

Physical properties, such as convex hull, were suggested to be one of the building blocks of numerical cognition ${ }^{1}$. Their role was demonstrated in comparative judgments of both symbolic $^{15}$ and non-symbolic comparison tasks ${ }^{2,3}$. However, it was never generalized to enumeration tasks, hence, it was unclear if the use of physical properties was specific to comparison tasks or if it was basic for general numerical abilities. Understanding the role of physical properties in other numerical abilities, such as enumeration, adds the missing link concerning their part in numerical cognition and strengthens the claim that physical properties are the corner stone of numerical cognition.

To conclude, we put forward the notion that a physical property can be used to downsample perceived information, enabling a heuristic ${ }^{10}$ for enumeration. Hence, we suggest that Jevons relied on the $\mathrm{CH}$ heuristic to estimate numerosity, similar to participants in many experiments that followed. 


\section{Methods}

Simulation. Probabilities $P(k \mid n)$ were estimated using a Monte Carlo simulation programmed in MATLAB. For every number $n$ between 3 and $30, n$ points were chosen randomly with a uniform distribution on the square $[0,1] \times[0,1]$. The number of vertices on the convex hull was then recorded, and the process was iterated $1,000,000$ times. For each $k$, the probability $P(k \mid n)$ was estimated as the fraction of times, out of the 1,000,000 iterations, where exactly $k$ vertices appeared on the convex hull of the $\mathrm{n}$ randomly chosen points. In order to ensure that the above process converges, the process was repeated. Two replications of the simulation deviated by at most 0.0015 , and in only 9 of the 378 relevant probabilities estimated did the deviation exceed 0.001 . Furthermore, for the cases where $P(k \mid n)$ is known from geometric theory, the results of the simulation deviated by at most 0.0007 from the known quantity, demonstrating that the simulation gives a good estimate of the probabilities.

Participants, sample size and informed consent. Sixteen participants (4 males, average age: 23.6 years old, SD: 1.31) took part in Experiment 1, and 36 (13 males, average age: 22.92 years old, SD: 1.63) took part in Experiment 2. All participants had normal or corrected-to-normal vision and received course credit for participation. The experiments were approved by the university ethics committee.

Stimuli. The stimuli were arrays of black dots on a white background. In the first experiment, all dots were the same size (diameter of 30 pixels). We used four numerosities (i.e. n's): 7, 8, 9, and 10; and five different $\mathrm{k}^{\prime} \mathrm{s}: 3,4,5,6$, and 7. In total, we had 20 conditions created by the combination of all chosen n's and k's. For each condition we presented 20 stimuli, resulting in 400 trials in total. In the second experiment, dot diameter varied between 10-69 pixels. We used fourteen numerosities (i.e. n's): 7-20 and three k's for each numerosity (see Table 1), that is, the $\mathrm{k}$ representing the most probable number of vertices and the $\mathrm{k}$ 's representing at least $30 \%$ more and $30 \%$ less than the most probable number of vertices on the convex hull. In total, we had 42 conditions created by the combination of all chosen n's and k's. For each condition we presented 10 stimuli, resulting in 420 trials in total.

Procedure. Participants were asked to estimate the number of dots presented on the screen. They were told the dots would appear for a very short duration so they could not count them, but needed to estimate. Each trial began with a black fixation point presented on a white screen for $1,000 \mathrm{~ms}$. This was followed by a blank white screen for another $1,000 \mathrm{~ms}$ and then the stimulus was presented for $16 \mathrm{~ms}$. After the stimulus was presented, the participants had 4,800 ms to respond vocally. After the participants responded, an experimenter coded the response. The experiment began with five practice trials. For Experiment 1, after the practice block, 400 trials were split into 4 blocks of 100 trials each. For Experiment 2, 420 trials were split to 4 blocks of 105 trials each. Participants' estimation and response times (RT) were recorded.

Analyses. Pre-processing: In both experiments, we removed trials in which participants gave estimations in the subitizing range (lower than 5). In Experiment 1, we removed trials in which participants gave estimations higher than 15 and trials in which they did not give a response, resulting in a total of 108 trials that were removed. In Experiment 2, we removed trials in which participants gave estimations of 40 and higher and trials in which they did not give a response, resulting in a total of 273 trials that were removed. 
Analysis: We ran a 2-way analysis of variance (ANOVA) with total numerosity and number of vertices on the convex hull as within subject variables, and subjects estimation as the dependent variable. In both experiments, assumption of sphericity was violated, however, results remained the same after correction. 


\section{References}

1. Leibovich, T., Katzin, N., Harel, M. \& Henik, A. From "sense of number" to "sense of magnitude": The role of continuous magnitudes in numerical cognition. Behav. Brain Sci. 40, e164 (2017).

2. Gebuis, T. \& Reynvoet, B. The interplay between nonsymbolic number and its continuous visual properties. J. Exp. Psych. Gen. 141, 642-648 (2012).

3. Leibovich, T. \& Henik, A. Comparing performance in discrete and continuous comparison tasks. Q. J. Exp. Psychol. 67, 899-917 (2014).

4. Kaufman, E. L., Lord, M. W., Reese, T. \& Volkmann, J. The discrimination of visual number. Am. J. Psychol. 62, 498-525 (1949).

5. Jevons, W. S. The power of numerical discrimination. Nature 3, 281-282 (1871).

6. Piazza, M., Mechelli, A., Butterworth, B. \& Price, C. J. Are subitizing and counting implemented as separate or functionally overlapping processes? Neurolmage 15, 435446 (2002).

7. Trick, L. M. \& Pylyshyn, Z. W. Why are small and large numbers enumerated differently? A limited-capacity preattentive stage in vision. Psychol. Rev. 101, 80-103 (1994).

8. Cowan, N. The magical number 4 in short-term memory: A reconsideration of mental storage capacity. Behav. Brain Sci. 26, 87-116 (2001).

9. Mandler, G. \& Shebo, B. J. Subitizing: an analysis of its component processes. J. Exp. Psychol. Gen. 111, 1-22 (1982).

10. Croft, H. T., Falconer, K. J. \& Guy, R.K. in Unsolved Problems in Geometry 6-47 (Springer, 1991).

11. Trott, M. in The Mathematical Guide Book for Symbolics 298-311 (Springer-Verlag, 2006). 
12. Valtr, P. Probability that $\mathrm{n}$ random points are in convex position. Discrete Comput. Geom. 13, 637-643 (1995).

13. Desimone, R. \& Duncan, J. Neural mechanisms of selective visual attention. Annu. Rev. Neurosci. 18, 193-222 (1995).

14. Gigerenzer, G. \& Gaissmaier, W. Heuristic decision making. Annu. Rev. Psychol. 62, 451482 (2011).

15. Henik, A. \& Tzelgov, J. Is three greater than five: The relation between physical and semantic size in comparison tasks. Mem. Cognit. 10, 389-395 (1982). 
CONVEX HULL AS A HEURSTIC, PREPRINT APRIL 2018

\section{Additional information.}

Correspondence and requests for materials should be addressed to N.K. naamaka@post.bgu.ac.il 